\section{TROMBOEMBOLISMO VENOSO: PROFILAXIA EM PACIENTES CLÍNICOS - PARTE II}

Autoria: Academia Brasileira de Neurologia, Associação de Medicina Intensiva Brasileira, Federação Brasileira das Associações de Ginecologia e Obstetrícia, Sociedade Brasileira de Angiologia e de Cirurgia Vascular, Sociedade Brasileira de Cancerologia, Sociedade Brasileira de Cardiologia, Sociedade Brasileira de Clínica Médica, Sociedade Brasileira de Geriatria e Gerontologia, Sociedade Brasileira de Hematologia e Hemoterapia, Sociedade Brasileira de Pneumologia e Tisiologia, Sociedade Brasileira de Reumatologia

Participantes: Ana Thereza Rocha, Edison Ferreira de Paiva, Arnaldo Lichtenstein, Rodolfo Milani Junior, Cyrillo Cavalheiro Filho, Francisco Humberto de Abreu Maffei, Charles André, Marcia Maiumi Fukujima, Gabriel R. de Freitas, Silvia Lage, Claudio Bonduki, Carlos Carvalho, Eduardo Ramacciotti, Vera Lúcia Teixeira, Paulo Roberto Mattos da Silveira, Clarissa Mathias, José Carlos Nicolau, Renato Delascio Lopes, Salo Buksman, Verônica Hagemeyer Santos, Élbio D’Amico, Mário Terra-Filho, Roger Abramino Levy, Alexandre Wagner Silva de Souza

Descrição do método de coleta de evidência: A pesquisa envolveu os seguintes bancos de dados: a) Medline (de 1966 a agosto de 2004), b) Base de Dados Cochrane de Revisões Sistemáticas (Cochrane Library), c) LILACS (de 1985 a 2004), d) resumos de estudos publicados em encontros científicos das sociedades envolvidas, e) referências cruzadas do material levantado. A estratégia utilizada no Medline foi ("Thrombosis"[MeSH] OR "Venous Thrombosis"[MeSH] OR "Embolism and Thrombosis"[MeSH] OR "Embolism"[MeSH] OR "Pulmonary Embolism"[MeSH]) NOT "Atrial Fibrillation"[MeSH] NOT ("Surgery" [MeSH] OR "surgery"[Subheading] OR "Surgical Procedures, Operative"[MeSH]) NOT ("Wounds and Injuries"[MeSH] OR "injuries"[Subheading] OR "Multiple Trauma"[MeSH])".

\section{Graus de recomendação e força de evidência:}

A: Estudos experimentais ou observacionais de melhor consistência.

B: Estudos experimentais ou observacionais de menor consistência.

C: Relatos de casos (estudos não controlados).

D: Opinião desprovida de avaliação crítica, baseada em consensos, estudos fisiológicos ou modelos animais.

\section{Introdução}

Tromboembolismo venoso (TEV) representa um espectro de doenças que inclui trombose venosa profunda, trombose associada a cateteres venosos centrais e, a complicação mais grave, tromboembolismo pulmonar (TEP). Tanto episódios assintomáticos quanto aqueles clinicamente aparentes em pacientes hospitalizados estão associados à fatalidade. Em hospitais brasileiros e de todo o mundo, a suspeita antemortem é feita em pequena proporção dos TEP. A ênfase dada à incidência de TEV em populações cirúrgicas despertou a necessidade de profilaxia para este grupo de pacientes. No entanto, mais da metade dos pacientes que sofrem TEV sintomático não têm doenças cirúrgicas, mas sim clínicas. Os fatores de risco para TEV em pacientes não submetidos à cirurgia e as medidas profiláticas são abordados nas diretrizes de profilaxia de TEV em pacientes clínicos, dividida em três partes. Na primeira parte, encontram-se as informações sobre: mobilidade reduzida, idade $>55$ anos, história prévia de TEV, varizes, insuficiência venosa crônica, insuficiência arterial periférica, obesidade, trombofilias hereditárias e adquiridas. $\mathrm{Na}$ segunda parte: gravidez, pós-parto, reposição hormonal, contraceptivos, insuficiência cardíaca congestiva classes III-IV, infarto agudo do miocárdio, acidente vascular cerebral isquêmico, acidente vascular cerebral hemorrágico, paresia e paralisia de membros inferiores. Na terceira parte: doença reumatológica ativa, doença inflamatória intestinal, doenças respiratórias graves, infecções com exceção das torácicas, síndrome nefrótica, câncer, quimioterapia, hormonioterapia, internação em unidade de terapia intensiva, uso de cateteres venosos centrais, uso de cateteres para hemodiálise, uso de Swan-Ganz. Versão integral e extensa que originou essas diretrizes encontra-se em documento elaborado pelo Grupo de Estudos de Trombose e Hemostasia. Para identificar os estudos, uma pesquisa auxiliada por computador foi feita, independentemente, por cada representante das sociedades e pelos coordenadores para cada um dos assuntos do parágrafo acima. Procurou-se, também, estudos avaliando a eficácia dos métodos de profilaxia contra TEV, nesta população, incluindo heparina não-fracionada (HNF), heparinas de baixo peso molecular (HBPM) e métodos físicos.

A seleção dos estudos foi baseada na leitura do título e do resumo e, quando necessário, na leitura do texto completo, por pelo menos dois investigadores de cada área. A avaliação da qualidade dos estudos baseou-se em roteiros padronizados, considerando-se: desenho do estudo, randomização, recrutamento de pacientes consecutivos, seguimento apropriado, método de detecção de TEV, avaliação mascarada, precisão dos resultados e aplicabilidade dos resultados às questões clínicas sobre fatores de risco ou eficácia da profilaxia contra TEV. Como se avaliou nesta revisão tanto o risco quanto a eficácia de profilaxia contra TEV como desfechos principais dos estudos, foram incluídos, preferencialmente, ensaios clínicos controlados e randomizados (ECCR), mas também estudos de coorte e caso-controle. Foram coletados: desenho do estudo, número de pacientes recrutados e analisados, características da população investigada, tipo de teste de triagem usado para detecção de TEV e incidência de TEV no grupo com o fator de risco, com e sem uso de profilaxia, se aplicável. Quando possível, foram também coletados ou calculados o risco relativo (RR) ou a razão de chances ("odds ratio" ou OR), a redução do risco relativo (RRR) e o número necessário para causar danos ("number needed to harm" ou NNH), para estudos investigando risco, e o número necessário tratar ("number needed to treat" ou NNT), para estudos investigando benefícios da profilaxia, e o intervalo de confiança (IC).

\section{Gravidez e pós-parto}

A gravidez constitui um estado de hipercoagulabilidade que está conhecidamente associado a aumento do risco de TEV, 
mesmo em pacientes ambulatoriais ${ }^{1}(\mathbf{B})$. Em estudo populacional envolvendo mais de 24.000 mulheres, a incidência estimada total de TEV, durante períodos de gravidez e pós-parto, foi de 103:100.000 (IC de 95\% 55-177) ${ }^{2}$ (B). A incidência caiu para 59:100.000 (IC de 95\% 24-121), quando se excluíram episódios de TEV relacionados a outros fatores de risco adquiridos. No entanto, para as gestações a termo, a incidência foi de 95:100.000, o que corresponde à incidência de TEV em pacientes com idade > 55 anos. Além disso, TEV fatal permanece como um dos problemas mais importantes entre pacientes grávidas e puerperais, sendo que existe um risco 10 vezes maior de morte entre aquelas com idade $>40 \operatorname{anos}^{3}(\mathbf{B})$. O benefício potencial de anticoagulação crônica para pacientes grávidas com história de complicações obstétricas e trombofilias tem sido motivo de debate $^{4}(\mathbf{D})$. Estudo do tipo revisão sistemática ${ }^{5}(\mathbf{A})$ encontrou apenas oito publicações sobre profilaxia para TEV durante a gravidez e o pós-parto, incluindo um total de 649 mulheres. Quatro dos estudos compararam métodos de profilaxia no período pré-natal: HBPM vs. HNF (Dois estudos), aspirina mais HNF vs. Apenas aspirina (Um estudo) e HNF vs. Nenhuma profilaxia (Um estudo). Quatro estudos avaliaram profilaxia pós-natal seguindo parto cesárea: um comparou hidroxietil-amido com HNF, dois compararam HNF ou HBPM com placebo e o último comparou HNF com HBPM. Devido à amostragem reduzida e ao fato de poucos estudos compararem os mesmos esquemas, não foi possível a avaliação da incidência de TEV e mortalidade, sendo a evidência insuficiente para permitir que se façam recomendações sobre profilaxia para TEV, durante a gravidez e o pós-parto, quando na ausência de trombofilias.

\section{Terapia de reposição hormonal e contracepção hormonal}

Estudo envolvendo 2.763 mulheres com coronariopatia observou um aumento do RR de 2,7 (IC de 95\% 1,4-5,0) para TEV e de 2,8 (IC de 95\% 0,9-8,7) para TEP entre as pacientes que receberam estrogênio mais progesterona ${ }^{6}(\mathbf{A})$. Outro estudo envolvendo 16.608 pacientes, randomizadas para receber estrogênio mais progesterona ou placebo, mostrou que pacientes no grupo tratamento apresentaram um RR de 2,11 (IC de 95\% 1,58-2,82) para TEV e de 2,13 (IC de 95\% 1,39-3,25) para TEP, quando comparadas àquelas no grupo $\operatorname{placebo}^{7}(\mathbf{A})$. Metanálise de estudo com diferentes delineamentos também mostrou que a TRH está associada a risco relativo de 2,14 (IC de 95\% 1,64-2,81) para o desenvolvimento de $\operatorname{TEV}^{8}(\mathbf{A})$. Além disto, o risco para o desenvolvimento de TEV é maior durante o primeiro ano após o início da terapia, especialmente em mulheres com história prévia de TEV. O risco estimado para TEV foi quatro vezes maior (IC de 95\% 1,9-8,3) para usuárias de TRH via oral, quando comparada à via transdérmica ${ }^{9}(\mathbf{B})$. Em um estudo multicêntrico, envolvendo 21 centros na Europa, África, Ásia e América Latina, 1.143 mulheres de 20 a 44 anos, com episódios de TEV, foram comparadas a 2.998 controles de idades correspondentes. Mostrou-se que o uso de CCH estava associado a um OR de 4,15 (IC de 95\% 3,09-5,57), em européias e de 3,25 (IC de 95\% 2,59-4,08), em mulheres dos países em desenvolvimento10(B). Embora o risco absoluto de TEV seja baixo, refletindo a população em que estes medicamentos são utilizados, existe um acréscimo global no risco de TEV em usuárias de $\mathrm{CCH}$ em comparação a não usuárias. O risco da ocorrência de TEV é maior no primeiro ano de utilização do contraceptivo ${ }^{11}(\mathbf{B})$. Metanálise recente mostrou que o risco da ocorrência de TEV é maior em usuárias de $\mathrm{CCH}$ de $3^{\mathrm{a}}$ geração (que contêm o progestogênio desogestrel ou o gestodeno) em comparação aos de $2^{a}$ geração (que contêm o progestogênio levonogestrel ou norgestrel) ${ }^{12}(\mathbf{A})$. Pacientes hospitalizadas por doenças clínicas, recebendo $\mathrm{TRH}$ ou $\mathrm{CCH}$, não foram especificamente incluídas em estudos de profilaxia para TEV. Apenas 63 pacientes com estas características foram randomizadas em um dos estudos e a relevância específica deste fator de risco isolado, no contexto do paciente clínico hospitalizado, ainda não foi estudada.

\section{Insuficiência cardíaca congestiva (ICC)}

A presença de ICC esteve associada significativamente ao desenvolvimento de TEV (OR 2,61, IC de 95\% 1,44-4,73) e, quanto menor a fração de ejeção (FE), maior a probabilidade de desenvolvimento de TEV (OR 38,3 para $\mathrm{FE}<20 \%$, 2,8 para $\mathrm{FE}$ entre $20 \%$ e $40 \%$ e 1,7 para FE $>45 \%$ ), em estudo caso-controle, com 790 pacientes ${ }^{13}(\mathbf{B})$. Mesmo em pacientes recebendo anticoagulação após episódio de TEV, a ICC continua a ser um fator de risco independente para o desenvolvimento de novos episódios (OR 2,27; IC de 95\% 1,08-4,97; $p=0,03)^{14}$ (B). Em 100 pacientes portadores de ICC e/ou infecção torácica, a utilização de HNF, na dose de 5.000 UI 8-8 horas, reduziu drasticamente a incidência de TVP de $26 \%$ para 4\% $(p<0,01)^{15}(\mathbf{A})$. A eficácia da HNF na profilaxia de TEV, em pacientes portadores de IAM, difere segundo a presença de ICC, a incidência de TEV foi de $57 \%$, nos pacientes que evoluíram com ICC vs. $22 \%$, nos pacientes que não evoluíram ${ }^{16}(\mathbf{B})$. Estudo randomizado aberto com 665 pacientes, sendo 333 portadores de ICC e 332 com doença respiratória, comparou HNF, na dose de 5.000 UI SC 8-8 horas, com enoxaparina $40 \mathrm{mg}$ SC uma vez ao dia e não houve diferença na incidência de TEV entre os dois grupos como um todo (8,4\% vs. $10,4 \%)$; entretanto, no subgrupo de pacientes com ICC, houve um maior benefício naqueles recebendo enoxaparina $(9,7 \%$ vs $16,1 \%$; one-sided $p=0,014)^{17}(\mathbf{A})$. Análise de subgrupo mostrou que a enoxaparina $40 \mathrm{mg}$ nos pacientes portadores de ICC classe III ou IV reduz de 14,6\%, no grupo placebo para 4,0\% a incidência de TEV (RRR 0,72; IC de 95\% 0,19-0,91; $p=0,02)^{18}(\mathbf{B})$.

\section{Infarto agudo do miocárdio (IAM)}

A maioria dos dados de incidência de TEV em pacientes com IAM provém de estudos mais antigos e mostram resultados bastante variáveis, com valores que vão de $10 \%$ até $62,5 \%$, naqueles infartados que permaneceram em repouso absoluto no leito por cinco dias. Não só TVP, mas também TEP são mais frequentes no paciente com IAM. Em estudo com 81 pacientes, que comparou HNF e placebo como estratégias profiláticas, observou-se uma incidência de TEP de $12,2 \%$, no grupo placebo (5/41) vs. 0/37, no grupo que recebeu $\operatorname{HNF}^{16}(\mathbf{A})$. Embora seja pequeno o número de pacientes portadores de IAM avaliados quanto à utilização de heparina na profilaxia de TEV contra placebo, a redução de risco absoluto a favor da heparina foi de $29,7 \%$ para 13,9\%, em um dos $\operatorname{estudos}^{19}(\mathbf{A})$, e de 195 para 
4\%, em outro ${ }^{20}(\mathbf{A})$. Em nossa revisão, não foram encontrados estudos recentes ou que avaliassem o uso da enoxaparina ou da nadroparina e, mesmo os que avaliaram a dalteparina ou a HNF foram pequenos ou utilizaram esquemas de dose diferentes das preconizadas em consensos internacionais para pacientes clínicos hospitalizados. É interessante ressaltar que a utilização de meia elástica de compressão gradual em uma das pernas do paciente, com a outra perna servindo como controle, obteve uma RRR de desenvolvimento de TVP de $89 \%^{21}(\mathbf{B})$. De qualquer modo, HNF e a dalteparina se mostraram eficazes na profilaxia destes pacientes, assim como a utilização de métodos físicos, como a deambulação precoce e MECG.

\section{Acidente vascular cerebral - AVCI}

Pacientes hospitalizados com AVCI e mobilidade reduzida apresentam incidência bastante elevada de TVP, acometendo usualmente o membro afetado ${ }^{22}(\mathbf{B})$. Em estudos analisando métodos para prevenção de TEV comparados com placebo ou sem profilaxia, a proporção de TEV em pacientes dos grupos controle, avaliados por diferentes métodos de rastreamento, oscila entre $28 \%{ }^{23}(\mathbf{A})$ e $75 \%{ }^{22}(\mathbf{B})$. Nestes pacientes, o risco permanece alto por longos períodos, já que muitos persistem com hemiplegia, mesmo após a alta. Além disso, a proporção de pacientes que desenvolvem TEV após AVC depende da gravidade da condição e da presença de outros fatores como desidratação ${ }^{24}(\mathbf{C})$. É importante notar que como ocorre nos demais pacientes clínicos hospitalizados, na maior parte dos casos, os episódios de TVP são subclínicos. Entretanto, embolia pulmonar pode afetar negativamente o prognóstico do paciente com AVC; em um estudo, 5\% das mortes precoces foram relacionadas a embolia pulmonar ${ }^{25}(\mathbf{B})$. Por estes motivos, AVC isquêmico deve ser considerado como fator de risco para TEV. Quanto à profilaxia, alguns estudos pequenos, em pacientes hospitalizados com AVC e mobilidade reduzida, demonstraram redução significativa da incidência de TEV com o uso de métodos profiláticos. Não existem grandes estudos multicêntricos que tenham abordado a profilaxia de TEV em pacientes com AVC. A HNF comparada ao placebo, para prevenção de TEV em 305 pacientes com AVC nas últimas 48 horas, mostrou redução de TVP avaliada por fibrinogênio marcado, redução de TEP em exame post-mortem $(p<0,01)$ e redução da mortalidade no grupo tratado $(p<0,05)^{26}(\mathbf{A})$. Meta-análise de nove ensaios clínicos, envolvendo HNF ou HBPM em pacientes com AVC, incluindo um ensaio em pacientes com AVCH, demonstrou redução significativa de TVP (RR 0,34; IC de 95\% 0,27-0,42, $\mathrm{p}<0,001$ ), mas não de TEP ou morte por todas as $\operatorname{causas}^{27}(\mathbf{A})$. Estudo de 19.435 pacientes com suspeita de AVCI para avaliar a eficácia e segurança de HNF (em doses de 5.000 UI ou 12.500 UI de 12-12h) e/ou aspirina mostrou, após 14 dias, que houve redução significativa de TEP nos pacientes alocados para HNF $(0,5 \% \text { vs. } 0,8 \% p<0,05)^{28}(\mathbf{A})$. Entretanto, houve um excesso de eventos hemorrágicos (AVCH e hemorragia extracraniana) no grupo de HNF em comparação ao grupo "não-heparina", como também entre o grupo alocado para HNF 12.500 UI em comparação com HNF 5.000 UI. Não houve diferenças significativas nas taxas de mortes em 14 dias ou em seis meses. As HBPM e heparinoides também têm sido avaliados na prevenção de
TEV em pacientes com AVCl. Estudo duplo-cego empregando a dalteparina ou placebo, em 60 pacientes com AVCI, mostrou que TVP, identificada por fibrinogênio marcado e confirmada por flebografia, foi observada mais frequentemente nos pacientes no grupo placebo do que no grupo dalteparina (50\% vs. $22 \%$, $p=0,05)^{29}(\mathbf{A})$. Metanálise de diversos desfechos associados ao tratamento com HBPM e heparinoides, envolvendo 2.855 pacientes com AVC, mostrou redução significativa de TVP (OR 0,27; IC de 95\% 0,08-0,96) e TEP sintomático (OR 0,34; IC de 95\% 0,17-0,69), mas também um aumento significativo de hemorragias extracranianas (OR 2,17; IC de 95\% $1,10-4,28)^{30}(\mathbf{A})$. Não houve diferença significativa na taxa de desfechos combinados (morte ou seqüela) entre os grupos de tratamento e placebo. Revisão sistemática comparando HNF com HBPM em pacientes com AVCI confirmado ou presumido, envolvendo 705 pacientes, quatro estudos utilizando danaparoide e um enoxaparina, observou 55 casos de TVP, nos 414 pacientes alocados para receber HBPM (13\%) em comparação com 65 casos nos 291 pacientes alocados para receber $\operatorname{HNF}(22 \%)^{31}(\mathbf{A})$. Esta diferença levou a um OR de 0,52 (IC de 95\% 0,35-0,79, p $=0,002$ ) associado ao uso de HBPM ou danaparoide. Entretanto, o número de eventos importantes como TEP, morte e hemorragia intra ou extracraniana foi pequeno demais em cada um dos grupos para predizer, com certeza, os benefícios e riscos destas intervenções. Além disso, não houve relato de eventos como recorrência de $\mathrm{AVCl}$ ou desempenho funcional dos sobreviventes. Uma vez que a preocupação com hemorragia entre pacientes com AVC é grande, métodos mecânicos de profilaxia de TEV também têm sido examinados como alternativas aos métodos farmacológicos. Dispositivos como CPI e MECG mostram resultados conflitantes sobre sua eficácia. A eficácia de MECG em 97 pacientes na fase aguda de AVC mostrou redução no risco de TVP, em geral, de $21,9 \%$ para $10,8 \%$, e de $6,3 \%$ para $4,6 \%$ em TVP proximal, mas a redução de TVP, em geral, não foi clinicamente significante (OR 0,43; IC de 95\% 0,14-1,36) ${ }^{32}(\mathbf{A})$. De 432 pacientes com $\mathrm{AVCl}$ e mobilidade reduzida que receberam HNF $5.000 \mathrm{UI}$ de 12-12h, MECG e CPI, comparados com 249 controles históricos recebendo HNF $5.000 \mathrm{UI}$ de 12-12h e MECG, um (0,23\%) paciente desenvolveu TVP e nenhum desenvolveu TEP, enquanto $9,2 \%$ dos controles desenvolveram TVP e 2,4\%, TEP. A adição de CPI ao esquema de profilaxia nestes pacientes de alto risco levou a uma redução de 40 vezes na incidência de $\operatorname{TVP}^{33}(\mathbf{B})$. Metanálise envolvendo 123 pacientes observou que MECG e CPI não foram associados a uma redução significativa na incidência de TVP, durante o período de tratamento dos sobreviventes de AVC, nem na mortalidade ${ }^{34}(\mathbf{A})$.

\section{Acidente vascular cerebral - AVCH}

Pacientes hospitalizados com AVCH podem ter uma incidência mais elevada de TEV em comparação com AVCI. Um estudo de coorte retrospectivo avaliou 1.953 pacientes com hemorragia craniana e 15.599 com isquemia cerebral, mostrando que a prevalência de TVP foi 4 vezes maior em pacientes com AVCH em comparação com AVCl. Mesmo após o controle de outros fatores de risco para TEV, como gravidade da condição e tempo de permanência no hospital, no AVCH, o risco (OR) para TVP 


\begin{tabular}{|c|c|c|}
\hline \multicolumn{3}{|c|}{$\begin{array}{l}\text { Evidência e recomeadaçōes especificas de profilaxia contra TEV em pacientes } \\
\qquad \text { som condį̧ø̄es clinicas đliversas. }\end{array}$} \\
\hline Condição & Mátndo & Dose \\
\hline $\begin{array}{l}\text { TRH/CCH+ } \\
\text { situą̧os de } \\
\text { risco }\end{array}$ & $\begin{array}{l}\text { HNF(D) } \\
\text { HBPM(D) }\end{array}$ & $\begin{array}{l}5.000 \text { Ui } 8-8 \pi \\
\text { Dalteparina ( } 5.000 \text { ul por dia) ou } \\
\text { ennoxapanna ( } 40 \mathrm{mg} g \mathrm{~d})\end{array}$ \\
\hline ICC & $\begin{array}{l}\text { HNF' }(\boldsymbol{A}) \\
\text { Enoxaparina'(A) } \\
\text { Dalteparina(D) } \\
\text { Nadroparina(D) }\end{array}$ & $\begin{array}{l}5.000 \text { Ul 8-81 } \\
40 \mathrm{mg} \text { por da } \\
5.000 \text { Uil por dia } \\
3.800 \text { Uil ( } 70 \mathrm{~kg}) \text { ou } 5.700 \mathrm{Ul}(270 \mathrm{~kg})\end{array}$ \\
\hline IAM & 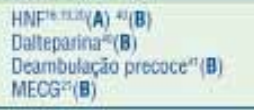 & $\begin{array}{l}5.000 \text { Ui } 12-12 \text { h ou dose plent } \\
120 \text { Ulikg }\end{array}$ \\
\hline \multirow[t]{2}{*}{ AVC isquêmico } & 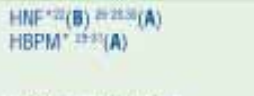 & $\begin{array}{l}\text { 5.000 Ui 8-8h ou } 12-12 \pi \\
\text { Dalteparina }(2500 \text { Ul } 12-12 \mathrm{~h}) \text { ou } \\
\text { enowagarina }(40 \mathrm{mg} / \mathrm{U})\end{array}$ \\
\hline & 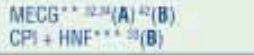 & $\mathrm{CPI}+\mathrm{HNF} 5.000$ UI $12-12 \mathrm{~h}$ \\
\hline AVC bemortágito & 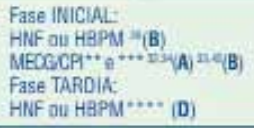 & NAOO USAR \\
\hline \multicolumn{3}{|c|}{ 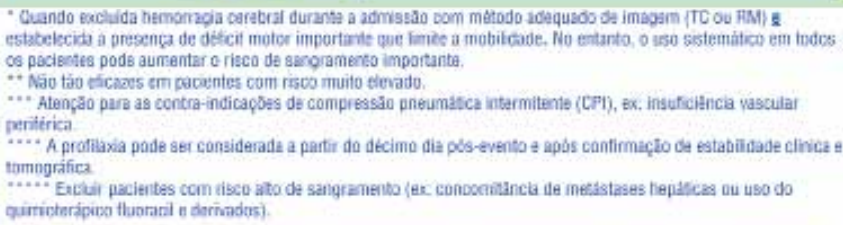 } \\
\hline \multicolumn{3}{|c|}{ 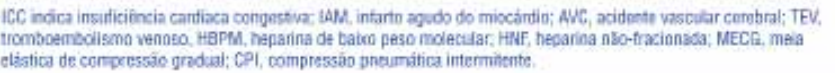 } \\
\hline
\end{tabular}

foi de 2,60; IC de 95\% 1,49-4,55; $(p=0.0008)^{35}(\mathbf{B})$. Dois pequenos estudos abordaram a utilização de profilaxia de TEV com heparina na fase aguda da hemorragia intracraniana, com resultados conflitantes em termos de eficácia, um deles não mostrando benefício ${ }^{36}(\mathbf{B})$ e o outro $\operatorname{sim}^{37}(\mathbf{B})$. Não foi, entretanto, verificado um excesso de complicações hemorrágicas. Devido à escassez de estudos conclusivos sobre profilaxia neste grupo de pacientes de alto risco para TEV não há evidência definitiva para sua recomendação, além do temor de sangramento importante em pacientes com AVCH. Paresia e Paralisia de Membros Inferiores Em um estudo com 143 pacientes que desenvolveram hemiplegia aguda, a incidência de TEV foi de $26 \%$ e o risco de TEV foi maior durante as primeiras quatro semanas do início da paralisia ${ }^{38}(\mathbf{B})$. Em estudo do tipo caso-controle com 620 pacientes idosos, paresia ou paralisia de membros inferiores estava independentemente associada a maior chance de TEV $\left(\right.$ OR 2,06) ${ }^{39}(\mathbf{B})$.

\section{Referências}

1. Samama MM. An epidemiologic study of risk factors for deep vein thrombosis in medical outpatients: the Sirius study. Arch Intern Med. 2000;160:3415-20.

2. Samuelsson E, Hagg S. Incidence of venous thromboembolism in young Swedish women and possibly preventable cases among combined oral contraceptive users. Acta Obstet Gynecol Scand. 2004;83:674-81.

3. Franks AL, Atrash HK, Lawson HW, Colberg KS. Obstetrical pulmonary embolism mortality, United States, 1970-85. Am J Public Health. 1990;80:720-2.

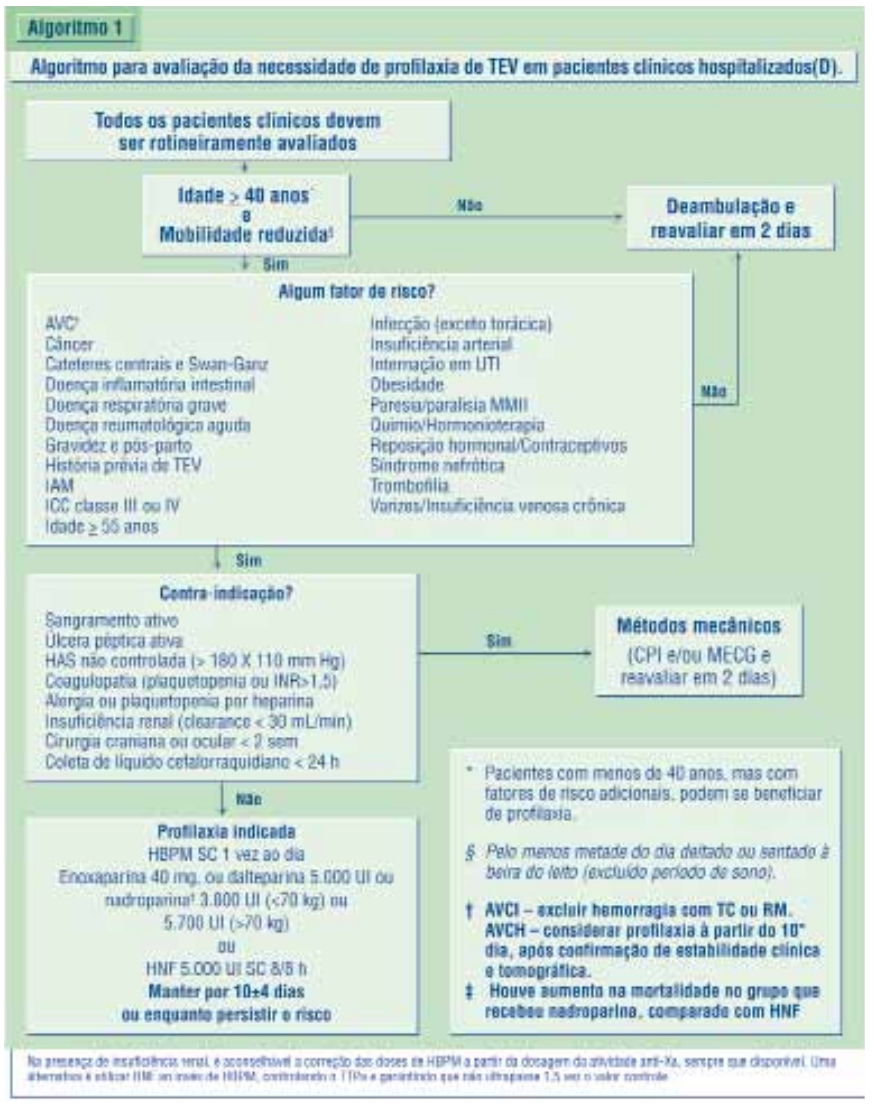

4. Brenner BR, Nowak-Gottl U, Kosch A, Manco-Johnson M, Laposata M. Diagnostic studies for thrombophilia in women on hormonal therapy and during pregnancy, and in children. Arch Pathol Lab Med. 2002;126:1296-303. 5. Gates S, Brocklehurst P, Davis L. Prophylaxis for venous thromboembolic disease in pregnancy and the early postnatal period. Cochrane Database Systematic Reviews; 2004.

6. Hulley S, Grady D, Bush T, Furberg C, Herrington D, Riggs B, et al. Randomized trial of estrogen plus progestin for secondary prevention of coronary heart disease in postmenopausal women. Heart and Estrogen/progestin Replacement Study (HERS) Research Group. JAMA. 1998;280:605-13.

7. Rossouw JE, Anderson GL, Prentice RL, LaCroix AZ, Kooperberg C, Stefanick ML, et al. Risks and benefits of estrogen plus progestin in healthy postmenopausal women: principal results From the Women's Health Initiative randomized controlled trial. JAMA. 2002;288:321-33.

8. Miller J, Chan BK, Nelson HD. Postmenopausal estrogen replacement and risk for venous thromboembolism: a systematic review and meta-analysis for the U.S. Preventive Services Task Force. Ann Intern Med. 2002;136:680-90. 9. Scarabin PY, Oger E, Plu-Bureau G. Differential association of oral and transdermal oestrogen-replacement therapy with venous thromboembolism risk. Lancet. 2003;362:428-32.

10. Venous thromboembolic disease and combined oral contraceptives: results of international multicentre case-control study. World Health Organization Collaborative Study of Cardiovascular Disease and Steroid Hormone Contraception. Lancet. 1995;346:1575-82.

11. Lidegaard O, Edstrom B, Kreiner S. Oral contraceptives and venous thromboembolism. A case-control study. Contraception. 1998;57:291-301. 12. Kemmeren JM, Algra A, Grobbee DE. Third generation oral contraceptives and risk of venous thrombosis: meta-analysis. BMJ. 2001;323:131-4. 
13. Howell MD, Geraci JM, Knowlton AA. Congestive heart failure and outpatient risk of venous thromboembolism: a retrospective, case-control study. J Clin Epidemiol. 2001;54:810-6.

14. Douketis JD, Foster GA, Crowther MA, Prins MH, Ginsberg JS. Clinical risk factors and timing of recurrent venous thromboembolism during the initial 3 months of anticoagulant therapy. Arch Intern Med. 2000;160:3431-6.

15. Belch JJ, Lowe GD, Ward AG, Forbes CD, Prentice CR. Prevention of deep vein thrombosis in medical patients by low-dose heparin. Scott Med J. 1981;26:115-7.

16. Emerson PA, Marks P. Preventing thromboembolism after myocardial infarction: effect of low-dose heparin or smoking. Br Med J. 1977;1:18-20.

17. Kleber FX, Witt C, Vogel G, Koppenhagen K, Schomaker U, Flosbach CW, et al. Randomized comparison of enoxaparin with unfractionated heparin for the prevention of venous thromboembolism in medical patients with heart failure or severe respiratory disease. Am Heart J. 2003;145: 614-21.

18. Alikhan R, Cohen AT, Combe S, SamamaMM, Desjardins L, Eldor A, et al. Prevention of venous thromboembolism in medical patients with enoxaparin: a subgroup analysis of the MEDENOX study. Blood Coagul Fibrinolysis. 2003;14:341-6.

19. Pitt A, Anderson ST, Habersberger PG, Rosengarten DS. Low dose heparin in the prevention of deep-vein thromboses in patients with acute myocardial infarction. Am Heart J. 1980;99: 574-8.

20. Zawilska K, Psuja P, Lewandowski K, Wroz M. Low-dose heparin in the prevention of thrombotic complications following acute myocardial infarction. Cor Vasa. 1989;31:179-85.

21. Kierkegaard A, Norgren L. Graduated compression stockings in the prevention of deep vein thrombosis in patients with acute myocardial infarction. Eur Heart J. 1993;14:1365-8.

22. McCarthy ST, Turner JJ, Robertson D, Hawkey CJ, Macey DJ. Low-dose heparin as a prophylaxis against deep-vein thrombosis after acute stroke. Lancet. 1977;2:800-1.

23. Turpie AG, Levine MN, Hirsh J, Carter CJ, Jay RM, Powers PJ, et al. Double-blind randomised trial of Org 10172 lowmolecular-weight heparinoid in prevention

of deep-vein thrombosis in thrombotic stroke. Lancet. 1987;1:523-6.

24. Kelly J, Hunt BJ, Lewis RR, Swaminathan R, Moody A, Seed PT, et al. Dehydration and venous thromboembolism after acute stroke. QJM. 2004;97:293-6.

25. Pambianco G, Orchard T, Landau P. Deep vein thrombosis: prevention in stroke patients during rehabilitation. Arch Phys Med Rehabil. 1995;76:324-30

26. McCarthy ST, Turner J. Low-dose subcutaneous heparin in the prevention of deep-vein thrombosis and pulmonary emboli following acute stroke. Age Ageing. 1986;15:84-8.

27. Sandercock PA, van den Belt AG, Lindley RI, Slattery J. Antithrombotic therapy in acute ischaemic stroke: an overview of the completed randomised trials. J Neurol Neurosurg Psychiatry. 1993;56:17-25.

28. The International Stroke Trial (IST): a randomised trial of aspirin, subcutaneous heparin, both, or neither among 19435 patients with acute ischaemic stroke. International Stroke Trial Collaborative Group. Lancet. 1997;349:1569-81.

29. Prins MH, Gelsema R, Sing AK, Van Heerde LR, den Ottolander GJ. Prophylaxis of deep venous thrombosis with a lowmolecular-weight heparin (Kabi 2165/Fragmin) in stroke patients. Haemostasis. 1989;19:245-50.

30. Bath PM, Iddenden R, Bath FJ. Lowmolecular-weight heparins and heparinoids in acute ischemic stroke: a meta-analysis of randomized controlled trials. Stroke. 2000;31:1770-8.

31. Counsell C, Sandercock P. Low-molecularweight heparins or heparinoids versus standard unfractionated heparin for acute ischaemic stroke. Cochrane Database Syst Rev. $2001 ;(4): C D 000119$.

32. Muir KW, Watt A, Baxter G, Grosset DG, Lees KR. Randomized trial of graded compression stockings for prevention of deep-vein thrombosis after acute stroke. QJM. 2000;93:359-64.

33. Kamran SI, Downey D, Ruff RL. Pneumatic sequential compression reduces the risk of deep vein thrombosis in stroke patients. Neurology. 1998;50:1683-8

34. Mazzone C, Chiodo GF, Sandercock P, Miccio M, Salvi R. Physical methods for preventing deep vein thrombosis in stroke. Cochrane Database Syst Rev. 2004;(4):CD001922.

35. Gregory PC, Kuhlemeier KV. Prevalence of venous thromboembolism in acute hemorrhagic and thromboembolic stroke. Am J Phys Med Rehabil. 2003;82:364-9.

36. Dickmann U, Voth E, Schicha H, Henze T, Prange H, Emrich D. Heparin therapy, deep-vein thrombosis and pulmonary embolism after intracerebral hemorrhage. Klin Wochenschr. 1988;66:1182-3.

37. Boeer A, Voth E, Henze T, Prange HW. Early heparin therapy in patients with spontaneous intracerebral haemorrhage. J Neurol Neurosurg Psychiatry. 1991;54:466-7.

38. Rentsch HP. Thromboembolism complications following acute hemiplegias. Schweiz Med Wochenschr. 1987;117:1853-5.

39. Weill-Engerer S, Meaume S, Lahlou A, Piette F, Saint-Jean O, Sachet A, et al. Risk factors for deep vein thrombosis in inpatients aged 65 and older: a case-control multicenter study. J Am Geriatr Soc. 2004;52:1299-304.

40. Scala PJ, Thiollet M, Midavaine M, Kher A, Funck-Brentano C, Jaillon P, et al. Deep venous thrombosis and left ventricular thrombosis prophylaxis by low molecular weight heparin in acute myocardial infarction. Haemostasis. 1990;20:368-9.

41. Miller RR, Lies JE, Carretta RF, Wampold DB, DeNardo GL, Kraus JF, et al. Prevention of lower extremity venous thrombosis by early mobilization. Confirmation in patients with acute myocardial infarction by $125 \mathrm{I}$-fibrinogen uptake and venography. Ann Intern Med. 1976;84:700-3.

42. Prasad BK, Banerjee AK, Howard H. Incidence of deep vein thrombosis and the effect of pneumatic compression of the calf in elderly hemiplegics. Age Ageing. 1982;11:42-4.

0 texto completo da Diretriz: Tromboembolismo Venoso: Profilaxia em Pacientes Clínicos - Parte II está disponível nos sites: www.projetodiretrizes.org.br e www.amb.org.br. 